\title{
Stimulation of gastrointestinal antibody to Shiga toxin by orogastric immunization in mice
}

\author{
MARK A. SUCKOW, ${ }^{1}$ DAVID F. KEREN,${ }^{2}$ J. EDWARD BROWN ${ }^{3}$ and \\ GERALD T. KEUSCH ${ }^{4}$
}

${ }^{1}$ Unit for Laboratory Animal Medicine, University of Michigan Medical School, ${ }^{2}$ Warde Medical Laboratory, Ann Arbor, Michigan, ${ }^{4}$ Division of Geographic Medicine and Infectious Diseases, Department of Medicine, New England Medical Center and Tufts University School of Medicine, Boston, Massachusetts, USA and ${ }^{3}$ Armed Forces Institute of Medical Sciences, Bangkok, Thailand

\begin{abstract}
Summary Shiga toxin (ST) is a protein toxin of Shigella dysenteriae type 1, a causative agent of severe diarrhoea and dysentery. In this report we describe the gastrointestinal secretory antibody response of mice following orogastric immunization with ST. Gastrointestinal secretions were sampled by a gastrointestinal lavage technique weekly for 5 weeks after initial immunization. Assay of lavage samples by ELISA showed that mice vaccinated orogastrically with various doses of ST developed gastrointestinal antibody to ST in a dose-dependent manner. Serum anti-ST activity developed by 5 weeks after initial immunization. The ability of ST to act as a mucosal immune adjuvant was investigated by coadministration of ST and keyhole limpet haemocyanin. In contrast to cholera toxin, a potent adjuvant, ST did not demonstrate adjuvant activity. The mouse gastrointestinal lavage model could be useful for further analysis of the cellular basis of ST immunogenicity.
\end{abstract}

Key words: cholera toxin, mice, Shiga, Shigella dysenteriae.

\section{Introduction}

The mucosal surfaces of the body, especially those lining the gastrointestinal, respiratory and genitourinary tracts, are continuously exposed to a wide variety of potentially pathogenic agents including viruses, bacteria, protozoa and toxins. In response, plasma cells in the mucosa are directed to synthesize and secrete antigen specific antibody. ${ }^{1}$ At mucosal surfaces, the predominant antibody isotype is secretory $\mathrm{IgA}$, while IgG predominates in serum. In either case, antibody can bind to and may inhibit harmful activity of encountered pathogenic agents.

Shiga toxin (ST) is a protein product of Shigella dysenteriae type 1 , a causative agent of severe diarrhoee and dysentery. ${ }^{2,3}$ In addition, ST has been implicated in the development of haemolytic uraemic syndrome. ${ }^{4-6}$ It is believed that ST may induce disease by damaging vascular endothelium. ${ }^{7-9}$

Although the exact role of ST in clinical disease remains undefined, the toxic effects of ST have been

Correspondence: M. A. Suckow, Laboratory Animal Program, Purdue University, South Campus Courts-D, West Lafayette, Indiana 47907, USA.

Received 12 January 1993; accepted 20 May 1993. described in several experimental models. The toxin is lethal for rabbits and mice when given parenterally, ${ }^{10,11}$ results in fluid accumulation in acutely ligated rabbit ileal loops, ${ }^{12,13}$ and is cytotoxic for various cell lines including human vascular endothelial cells. ${ }^{12,14-16}$ ST appears to act by binding of a proteolytically generated subunit to the $60 \mathrm{~S}$ ribosome with subsequent inhibition of protein synthesis and cell death. ${ }^{17}$

ST is structurally similar to cholera toxin (CT), a powerful adjuvant for the mucosal immune system, in that both are multimeric proteins composed of five B subunits which presumably bind to susceptible cells and a single A subunit believed responsible for generating a toxic subunit following endocytosis. ${ }^{18,19}$

Systemic humoral immunity to ST is readily stimulated and immune sera from humans and rabbits can neutralize in vitro activity of the toxin. ${ }^{20,21}$ The protective value of serum anti-ST antibody is questionable as demonstrated by failure of high circulating anti-ST titres to protect Rhesus monkeys against clinical shigellosis when orally challenged with live Shigella dysenteriae. ${ }^{22}$ Although these studies clearly describe the serum antibody response to ST, there is a lack of data describing local immunity in the gastrointestinal 
tract. Studies in rabbits have shown that, like CT, ST stimulates a powerful antigen specific secretory IgA response when administered directly into Thiry-Vella ileal loops. ${ }^{12}$ Furthermore, anti-ST secretory IgA inhibits HeLa cell cytotoxicity and fluid accumulation in acutely ligated rabbit ileal segments. ${ }^{12}$

Precise definition of the cellular basis for stimulation of the gastrointestinal antibody response to ST has been hampered by the expense and effort of using the rabbit Thiry-Vella ileal loop model, the lack of inexpensive inbred lines of rabbits, and the lack of readily available monoclonal antibodies to rabbit lymphocyte antigens. In this regard, an easily manipulated mouse model to study the mucosal immune response to ST would be useful. Described here is the application of a mouse orogastric lavage technique to investigate dose sensitivity of the gastrointestinal anti-ST secretory IgA response, development of this response over time, and studies to determine if ST, like CT, can exert an adjuvant effect for a weak mucosal protein antigen, keyhole limpett haemocyanin $(\mathrm{KLH}){ }^{23}$

\section{Materials and methods}

\section{Immunogens}

CT was obtained from List Biological Laboratories Inc. (Campbell, CA, USA). Purified ST used for immunization of mice was prepared by affinity column chromatography as described in detail elsewhere. ${ }^{24}$ Purified ST used as antigen in ELISA assays was prepared from the ammonium sulfate precipitate of culture lysates from $S$. dysenteriae by using a combination of ionexchange chromatography, gel filtration chromatography and preparative isoelectric focusing. ${ }^{25}$

\section{Animals}

Eight to twelve week old C57B1/6J female mice from the Jackson Laboratory (Bar Harbor, ME, USA) were used in all animal studies. All mice were specific pathogen free and were housed under conditions suitable to maintenance of a constant microbiologic status. Use of these animals was approved by the institutional animal care and use committee.

\section{Immunizations}

To examine the dose sensitivity of the gastrointestinal secretory $\mathrm{IgA}$ response to $\mathrm{ST}$, groups of five mice were dosed orogastrically with $0.01,0.10,1.0,10.0,25.0$ or $50.0 \mu \mathrm{g}$ of ST in $0.5 \mathrm{~mL}$ of $0.2 \mathrm{~mol} / \mathrm{L} \mathrm{NaHCO}_{3}$ via a 22 gauge stainless steel feeding needle at days $0,7,14$ and 21.

To examine the adjuvant activity of ST, groups of five mice were dosed orogastrically as described above with $0.5 \mathrm{~mL}$ of $0.2 \mathrm{~mol} / \mathrm{L} \mathrm{NaHCO}$ solutions containing $5 \mathrm{mg}$ KLH (Sigma Chemical Co., St Louis, MO, USA), $5 \mathrm{mg} \mathrm{KLH}$ and $10 \mu \mathrm{g} \mathrm{CT}, 5 \mathrm{mg} \mathrm{KLH}$ and $10 \mu \mathrm{g}$ ST, $10 \mu \mathrm{g} \mathrm{CT}$ or $10 \mu \mathrm{g} \mathrm{ST}$. In addition, one cohort was dosed with only $0.5 \mathrm{~mL}$ of $0.2 \mathrm{~mol} / \mathrm{L} \mathrm{NaHCO}_{3}$.

\section{Collection of gastric lavage samples}

Gastrointestinal contents were sampled prior to immunization and then weekly for 5 weeks following initial immunization by a previously described orogastric lavage technique. ${ }^{26}$ Briefly, mice were orogastrically dosed via a 22 gauge stainless steel feeding needle four times, $15 \mathrm{~min}$ apart, with $0.5 \mathrm{~mL}$ of a hyperosmotic solution consisting of $0.025 \mathrm{~mol} / \mathrm{L} \mathrm{NaCl}, 0.040 \mathrm{~mol} / \mathrm{L}$ $\mathrm{Na}_{2} \mathrm{SO}_{4}, 0.01 \mathrm{~mol} / \mathrm{L} \mathrm{KCl}, 0.002 \mathrm{~mol} / \mathrm{L} \mathrm{NaHCO}$ and $0.0485 \mathrm{~mol} / \mathrm{L}$ polyethylene glycol. Thirty minutes after the last lavage, each mouse received $0.5 \mathrm{~mL}$ of lactated Ringer's solution subcutaneously and $1.0 \mathrm{~mL}$ of $0.1 \%$ $(\mathrm{w} / \mathrm{v})$ pilocarpine intraperitoneally. Intestinal secretions were collected over the next $60 \mathrm{~min}$ in $3 \mathrm{~mL}$ of $0.1 \%(\mathrm{w} / \mathrm{v})$ soybean trypsin inhibitor. The samples were diluted to $6 \mathrm{~mL}$ with PBS and centrifuged at $600 \mathrm{~g}$ for $10 \mathrm{~min}$ to remove gross faecal debris. Thirty microlitres of $17 \%(\mathrm{w} / \mathrm{v})$ phenylmethylsulfonylfluoride (PMSF) in 95\% (v/v) ethanol was added to the supernatant prior to centrifuging at $27000 \mathrm{~g}$ for $20 \mathrm{~min}$ at $4{ }^{\circ} \mathrm{C}$. Next, $1 \%$ sodium azide $(\mathrm{w} / \mathrm{v})$ and PMSF were added to the resulting supernatant. After incubating $15 \mathrm{~min}$ at room temperature, $50 \mu \mathrm{L}$ of fetal calf serum $/ \mathrm{mL}$ of sample were added and the samples were frozen at $-20^{\circ} \mathrm{C}$.

\section{Collection of serum samples}

Blood samples were collected by retro-orbital bleeding of mice under methoxyfluorane anaesthesia (Metofane, Pitman-Moore, Washington Crossing, NJ, USA) prior to immunization and 5 weeks after initial immunization. Sera were stored at $-20^{\circ} \mathrm{C}$.

\section{Enzyme-linked immunosorbent assay}

Sera and gastrointestinal lavage samples were assayed in duplicate for anti-KLH, and anti-ST IgA, IgG and IgM via an ELISA technique. Polystyrene microdilution wells were coated with either KLH $(1.0 \mu \mathrm{g} /$ well $)$ in $200 \mathrm{mmol} / \mathrm{L}$ carbonate buffer (pH 9.6), or pure ST 
( $25 \mathrm{ng} /$ well) in carbonate buffer. Following overnight incubation, the plates were washed three times with PBS, pH 7.4, containing $0.1 \%(\mathrm{v} / \mathrm{v})$ Tween 20, and post-coated with $3 \%(\mathrm{w} / \mathrm{v})$ bovine serum albumin (Sigma Chemical Co., St Louis, MO, USA). Following removal of unbound protein by washing with PBS/ Tween, the plates were stored at $4^{\circ} \mathrm{C}$ for up to 2 weeks. Protein concentrations of gastrointestinal lavage and serum samples were measured by the Bradford method and standardized by dilution with PBS. Immediately prior to addition of serum or intestinal secretions, the antigen solutions were removed and the wells were again washed with PBS/Tween 20. Samples to be assayed were diluted in this buffer $(1: 2$ for intestinal lavage samples and $1: 10$ for sera), added to wells $(100 \mu \mathrm{L} /$ well $)$ and incubated for $12 \mathrm{~h}$. Following this incubation, the wells were washed with the buffer and incubated overnight at $4{ }^{\circ} \mathrm{C}$ with $1: 1000(\mathrm{v} / \mathrm{v})$ solutions containing either alkaline phosphatase conjugated rabbit anti-mouse IgG, alkaline phosphatase conjugated rabbit anti-mouse IgM or alkaline phosphatase conjugated goat anti-mouse IgA (Zymed Inc., South San Francisco, CA, USA) in PBS/Tween 20.

Monospecificity of IgG and IgM antisera was confirmed with ELISA. Slight anti-IgM activity in the antiIgA reagent was adsorbed out by adding 1:2000 (v/v) mouse IgM (Sigma Chemical, St Louis, MO, USA). For anti-KLH assays, plates underwent an additional wash with buffer and the substrate reaction was carried out with $0.1 \mathrm{~mL}$ of $p$-nitrophenyl phosphate $(1 \mathrm{mg}$ / $\mathrm{mL}$ ) in carbonate buffer at a constant temperature of $25^{\circ} \mathrm{C}$. The kinetics of the enzyme-substrate reaction were measured to $100 \mathrm{~min}$ on a Titertek Multiscan MicroELISA Reader (Flow Laboratories Inc., McLean, VA, USA) at an optical density of $405 \mathrm{~nm}$ (OD 405). For the anti-ST assay, an ELISA Amplification System (Bethesda Research Laboratories, Gaithersburg, MD, USA) was used in which plates underwent an initial reaction with nicotinamide adenine dinucleotide phosphate (NADPH) followed by reaction with iodonitrotetrazolium violet (INT-violet). Final incubation with substrate was carried out at a constant temperature of $25^{\circ} \mathrm{C}$. Optical density at $490 \mathrm{~nm}$ (OD 490) of the reaction was measured $30 \mathrm{~min}$ after addition of substrate.

\section{Statistical analysis}

Means and standard errors of ELISA absorbance values were compared using the Wilcoxon rank sum test. Statistical significance was reached when $P<0.05$.

\section{Results}

\section{Mucosal immune response to $S T$}

Mice inoculated orogastrically with ST mounted a strong dose-dependent intestinal anti-ST secretory IgA response (Fig. 1). Two weeks following initial inoculation, mice dosed with $1.0-25 \mu \mathrm{g}$ of ST began to demonstrate significantly elevated anti-ST SIgA titres which continued to increase through the fourth and fifth weeks.

A weak anti-ST IgG response was detected in the intestinal secretions (data not shown), however anti-ST IgM activity was not found. No blood was grossly visible in any of the samples. Control mice dosed with $\mathrm{NaHCO}_{3}$ did not develop significant anti-ST antibody activity (data not shown).

Four of five animals dosed with $50 \mu \mathrm{g}$ of ST died within 2 days after the initial immunization, presumably due to toxic effects of ST. The single surviving animal from this cohort had a weak anti-ST response.

\section{Serum antibody response to orally administered ST}

Five weeks following orogastric administration of $0.1 \mu \mathrm{g}$ or greater doses of ST, serum anti-ST IgG activity increased markedly (Fig. 2). This activity was significantly greater than pre-immunization anti-ST activity in groups dosed with $1.0 \mu \mathrm{g}$ or more of ST. Immunization with at least $1.0 \mu \mathrm{g}$ of ST also stimulated weak serum IgM and IgA anti-ST activity (data not

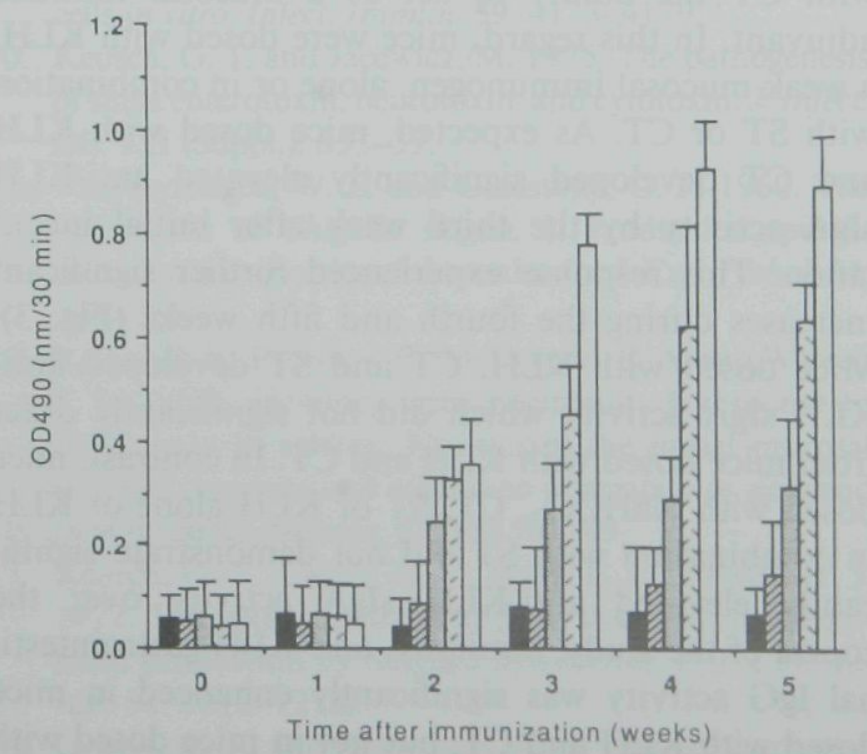

Fig. 1. Gastrointestinal anti-ST IgA activity for 5 weeks following initial immunization (week 0). Dose groups are

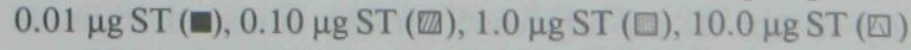
and $25.0 \mu \mathrm{g} \mathrm{ST}(\square)$. 


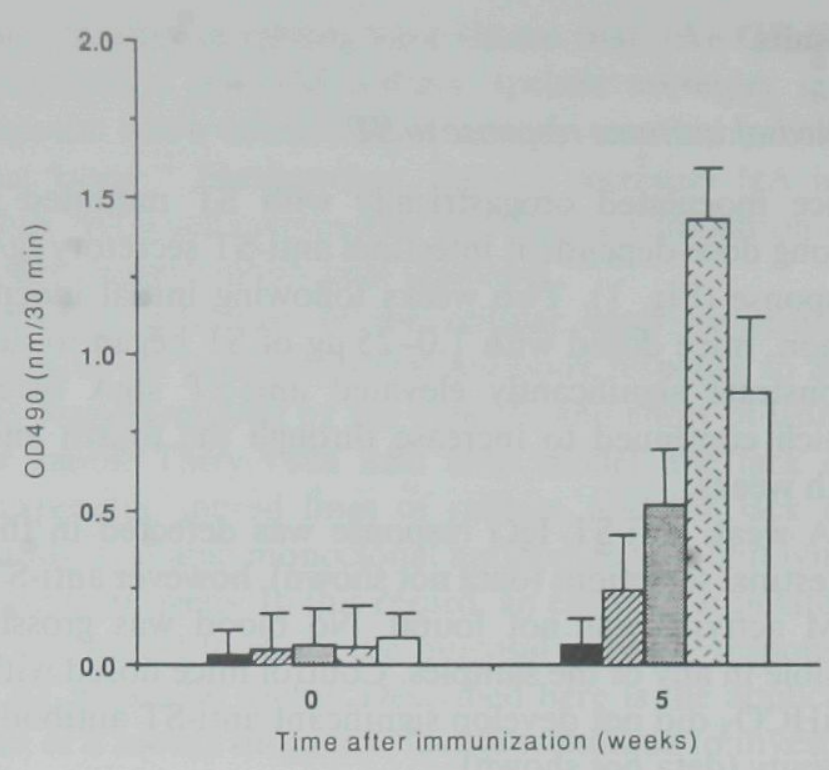

Fig. 2. Serum anti-ST IgG activity prior to immunization and at the time of killing ( 5 weeks after initial immunization). Dose groups are $0.01 \mu \mathrm{g}$ ST $(\mathbf{\square}), 0.10 \mu \mathrm{g}$ ST $(\mathbb{U}), 1.0 \mu \mathrm{g}$ ST $(\square), 10.0 \mu \mathrm{g} \mathrm{ST}(\square)$ and $25.0 \mu \mathrm{g} \mathrm{ST}(\square)$.

shown). Mice dosed with $0.01 \mu \mathrm{g}$ of ST did not mount a serum anti-ST response. Interestingly, mice dosed with $25 \mu \mathrm{g}$ of ST mounted a significantly weaker serum IgG anti-ST response than those dosed with $10 \mu \mathrm{g}$.

\section{Role of ST as a mucosal immune adjuvant}

Having established ST as a strong mucosal immunogen in the mouse, we examined whether ST shared with $\mathrm{CT}$ the ability to act as a mucosal immune adjuvant. In this regard, mice were dosed with $\mathrm{KLH}$, a weak mucosal immunogen, alone or in combination with ST or CT. As expected, mice dosed with $\mathrm{KLH}$ and $\mathrm{CT}$ developed significantly elevated anti-KLH sIgA activity by the third week after initial inoculation. This response experienced further significant increases during the fourth and fifth weeks (Fig. 3). Mice dosed with KLH, CT and ST developed antiKLH SIgA activity which did not significantly differ from mice dosed with $\mathrm{KLH}$ and $\mathrm{CT}$. In contrast, mice dosed with $\mathrm{NaHCO}_{3}, \mathrm{CT}$, ST or KLH alone or KLH in combination with ST did not demonstrate significantly elevated anti-KLH sIgA activity over the course of the study. Similarly, anti-KLH gastrointestinal IgG activity was significantly enhanced in mice dosed with KLH and CT, but not in mice dosed with $\mathrm{KLH}$ alone or in combination with ST (data not shown). These results indicate that ST lacks the ability to act as an adjuvant for the mucosal immune response to KLH.

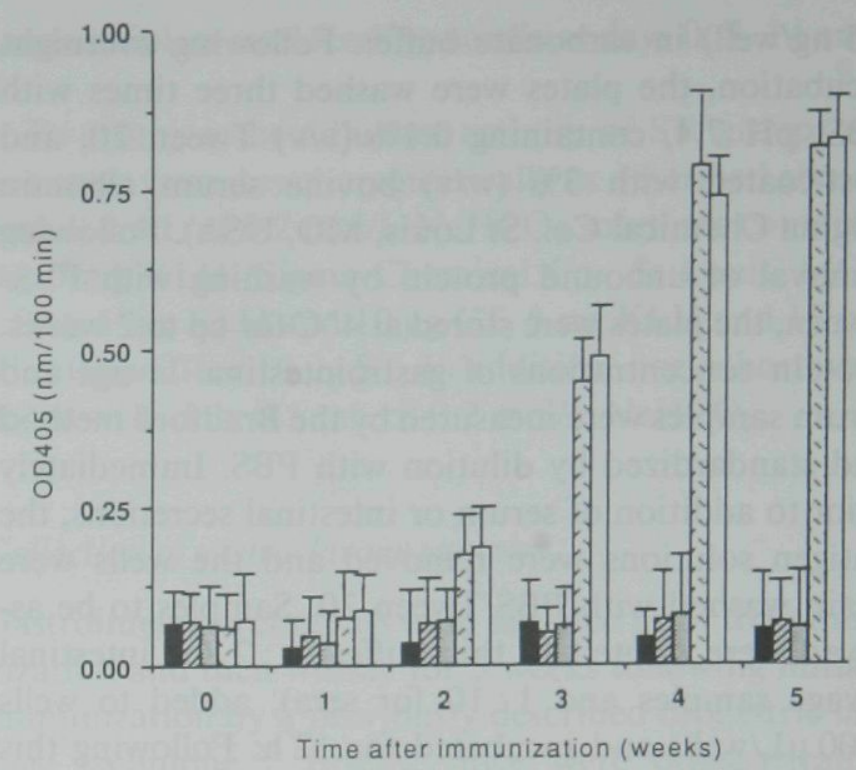

Fig.3. Gastrointestinal anti-KLH IgA activity through 5 weeks following initial immunization (week 0). Dose groups are $\mathrm{NaHCO}_{3}(\mathbf{\square}), \mathrm{KLH}(\mathbb{\pi}), \mathrm{KLH}+\mathrm{ST}(\square), \mathrm{KLH}+\mathrm{CT}$ $(\square)$, and $\mathrm{KLH}+\mathrm{ST}+\mathrm{CT}(\square)$.

\section{Discussion}

ST is likely an important virulence factor of Shigella dysenteriae, the causative agent of human dysentery. Like CT, a powerful mucosal antigen and adjuvant, ST elicits a strong secretory $\operatorname{IgA}$ response to itself following administration into rabbit Thiry-Vella ileal loops. ${ }^{12,23,27}$ The purpose of the present study was to develop a mouse model in which to study gastrointestinal immunity to ST and to examine the time course of the anti-ST gastrointestinal antibody response following orogastric immunization.

Anti-ST intestinal IgA and IgG activity developed in a dose-dependent manner in response to orogastric immunization with ST. Previous studies conducted in the rabbit chronic Thiry-Vella ileal loop model found that anti-ST sIgA activity is detectable by ELISA by the first week following direct intraloop immunization and increases after the second and third weekly doses. We found that in the mouse intestinal lavage model similar activity is detectable by the second week following orogastric immunization with ST. It is interesting that IgG directed against ST was found in gastrointestinal lavage samples following immunization. Although no blood was grossly observable in these samples, it is possible that small amounts of undetected blood were present. Alternatively, pilocarpine could result in transepithelial transport of serum components such as $\mathrm{IgG}$, since cholinergic agents have been shown to cause transient opening of tight junctions in the gut. ${ }^{28}$ 
Interestingly, mice dosed with $25 \mu \mathrm{g}$ of ST had lower serum anti-ST IgG activity than mice immunized with $10.0 \mu \mathrm{g} \mathrm{ST}$. A possible explanation might be the development of systemic tolerance to ST. Systemic tolerance following orogastric exposure has been described for several protein antigens. ${ }^{29-31}$ For many antigens, larger doses result in a greater and longer-lasting state of tolerance.

The mouse intestinal lavage model holds several advantages over the rabbit Thiry-Vella ileal loop model for the study of the mucosal antibody response to ST. First, greater numbers of animals can be studied using the mouse model, since it is less expensive and cumbersome than the surgically manipulated rabbit model. Second, the availability and use of inbred strains of mice diminish individual variation, due to genetic heterogeneity, in response to antigens. Third, commercial monoclonal antibodies to specific mouse $\mathrm{T}$-cell antigens and cytokines are readily available and will permit a thorough investigation of the cellular basis of ST immunogenicity. Fourth, the oral route of antigen administration more closely mimics the natural route of antigen exposure than exposure through a surgically created, isolated intestinal loop system.

Previous investigators have described the ability of $\mathrm{CT}$ to enhance the mucosal antibody response to a variety of unrelated antigens. ${ }^{27,32}$ The exact mechanism by which CT exerts this effect is unknown. Although several studies have suggested that adjuvanticity occurs through a cAMP independent pathway, other evidence suggests that this effect may be mediated through elevation of intracellular cAMP. ${ }^{33-36}$ In the present study, ST was unable to enhance the mucosal antibody response to a weak, coadministered, protein antigen (i.e. KLH). These results show that although ST is similar to CT in that it is both a strong immunogen and a multimeric enteric bacterial toxin, it may not be an adjuvant for the mucosal immune system. Further studies will be needed to determine if ST demonstrates adjuvancy for antigens other than KLH.

In conclusion, ST is a potent oral immunogen in mice, but lacks adjuvanticity. Although the precise role of ST in clinical disease remains largely speculative, the antigenicity of ST following orogastric immunization warrants further study to examine the potential usefulness of ST in immunization strategies to protect against Shigella-associated disease. Establishment of the mouse as an animal model in which to study gastrointestinal immunity to ST will permit a more detailed examination of the cellular basis of this response.

\section{References}

1. Bienenstock, J. E., Befus, A. D. and McDermott, M. 1981. Mucosal immunity. In The Mucosal Immune System, F. J. Bourne (ed.). Martinus Nijhoff Publishers, The Hague, pp. 5-27.

2. Donohue-Rolfe, A. and Keusch, G. T. 1983. Shigella dysenteriae 1 cytotoxin: Periplasmic protein releasable by polymyxin B and osmotic shock. Infect. Immun. 39: 270-274.

3. Griffin, D. K. and Gemski, P. 1983. Release of shiga toxin from Shigella dysenteriae 1 by polymyxin B. Infect. Immun. 40: 425-428.

4. Gianantonio, C., Vitacco, M., Mendilaharzu, F., Rutty, A. and Mendilaharzu, J. 1964. The hemolytic-uremic syndrome. J. Pediatr. 64: 478-491.

5. Koster, F., Levin, J., Walker, L. et al. 1977. Hemolyticuremic syndrome after shigellosis. Relation to endotoxemia and circulating immune complexes. N. Engl. J. Med. 298: 927-933.

6. Raghupathy, P., Date, A., Shastry, J. C., Sudarsanam, A. and Jadhav, M. 1978. Haemolytic-uraemic syndrome complicating shigella dysentery in south Indian children. Br. Med. J. 1: 1518-1521.

7. Tesh, V. L. and O'Brien, A. D. 1991. The pathogenic mechanisms of shiga toxin and the shiga-like toxins. Mol. Microbiol. 5: 1817-1822.

8. Tesh, V. L., Samuel, J. E., Perera, L. P., Sharefkin, J. B. and O'Brien, A. D. 1991. Evaluation of the role of shiga and shiga-like toxins in mediating direct damage to human vascular endothelial cells. J. Infect. Dis. 164: 344 352.

9. Louise, C. B. and Obrig, T. G. 1991. Shiga toxinassociated hemolytic-uremic syndrome: Combined cytotoxic effects of shiga toxin, interleukin-1, and tumor necrosis factor alpha on human vascular endothelial cells in vitro. Infect. Immun. 59: 4173-4179.

10. Keusch, G. T. and Jacewicz, M. 1975. The pathogenesis of shiga enterotoxin, neurotoxin, and cytotoxin. J. Infect. Dis. 131 (Suppl.): S33-39.

11. VanHeyningen, W. E. and Gladstone, G. P. 1960. The neurotoxin of Shigella shigae. I. Production, purification, and properties of the toxin. Br. J. Exp. Pathol. 34: 202-216.

12. Keren, D. F., Brown, J. E., McDonald, R. A. and Wassef, J. W. 1989. Secretory immunoglobulin A response to shiga toxin in rabbits: Kinetics of the initial mucosal immune response and inhibition of toxicity in vitro and in vivo. Infect. Immun. 57: 1885-1889.

13. Keusch, G. T., Grady, G. F., Mata, L. J. and McIver, J. 1972. The pathogenesis of Shigella diarrhea. 1. Enterotoxin production by Shigella dysenteriae 1. J. Clin. Invest. 51: 1212-1218.

14. Gentry, M. K. and Dalrymple, J. M. 1980. Quantitative microtiter cytotoxicity assay for Shigella toxin. J. Clin. Microbiol. 12: 361-366.

15. O'Brien, A. D. and LaVeck, G. D. 1982. Immunochemical and cytotoxic activities of Shigella dysenteriae 
1 (shiga) and shiga-like toxins. Infect. Immun. 35: 11511154.

16. Obrig, T. G., Del Vecchio, P. J., Brown, J. E. et al. 1988. Direct cytotoxic action of shiga toxin on human vascular endothelial cells. Infect. Immun. 56: 2373-2378.

17. Keusch, G. T., Donohue-Rolfe, A. and Jacewics, M. 1982. Shigella toxin(s): Description and role in diarrhea and dysentery. Pharmacol. Ther. 15: 403-438.

18. Donohue-Rolfe, A., Keusch, G. T., Edson, C., ThorleyLawson, D. and Jacewicz, M. 1984. Pathogenesis of Shigella diarrhea. IX. Simplified high yield purification of Shigella toxin and characterization of subunit composition and function by the use of subunit-specific monoclonal and polyclonal antibodies. J. Exp. Med. 160: 1767-1781.

19. Seidah, N. G., Donohue-Rolfe, A., Lazure, C., Auclair, F., Keusch, G. T. and Chretein, M. 1986. Complete amino acid sequence of Shigella toxin B-chain. A novel polypeptide containing 69 amino acids and one disulfide bridge. J. Biol. Chem. 261: 13928-13931.

20. Keusch, G. T. and Jacewicz, M. 1973. Serum enterotoxin-neutralizing antibody in human shigellosis. Nature New Biol. 241: 31-32.

21. Keusch, G. T., Jacewicz, M., Levine, M. M., Hornick, R. B. and Kochwa, S. 1976. Pathogenesis of Shigella diarrhea. Serum anticytotoxin antibody response produced by toxigenic and nontoxigenic Shigella dysenteriae $1 . J$. Clin. Invest. 57: 194-202.

22. McIver, J., Grady, G. F. and Formal, S. B. 1977. Immunization with Shigella dysenteriae type 1: Evaluation of antitoxic immunity in prevention of experimental disease in rhesus monkeys (Macaca mulatta). J. Infect. Dis. 156: $416-421$

23. Elson, C. O. and Ealding, W. 1984. Generalized systemic and mucosal immunity in mice after mucosal stimulation with cholera toxin. J. Immunol. 132: 2736-2742.

24. Donohue-Rolfe, A., Acheson, D. W., Kane, A. V. and Keusch, G. T. 1989. Purification of shiga toxin and shiga-like toxins I and II by receptor analog affinity chromatography with immobilized P1 glycoprotein and production of cross-reactive monoclonal antibodies. Infect. Immun. 57: 3888-3893.

25. Brown, J. E., Griffin, D. E., Reitman, S. W. and Doctor, B. P. 1982. Purification of shiga toxin from Shigella dysenteriae 1. Infect. Immun. 36: 996-1005.

26. Elson, C. O., Ealding, W. and Lefkowitz, J. 1984. A lavage technique allowing repeated measurement of IgA antibody in mouse intestinal secretions. J. Immunol. Meth. 67: 101-108.

27. Lycke, N. and Holmgren, J. 1986. Strong adjuvant properties of cholera toxin on gut mucosal immune responses to orally presented antigens. Immunol. 59: 301308.

28. Phillips, T. E., Phillips, T. L. and Neutra, M. R. 1987. Macromolecules can pass through occluding junctions of rat ileal epithelium during cholinergic stimulation. Cell Tissue Res. 247: 547-554.

29. Richman, L. K., Chiller, J. M., Brown, W. R., Hanson, D. G. and Maz, N. M. 1978. Enterically induced immunologic tolerance. I. Induction of suppressor T lymphocytes by intragastric administration of soluble proteins. J. Immunol. 121: 2429-2436.

30. Ngan, J. and Kind, L. S. 1978. Suppressor T cells for IgE and IgG in Peyer's patches of mice made tolerant by the oral administration of ovalbumin. J. Immunol. 120: 861-869.

31. Miller, S. D. and Hanson, D. G. 1979. Inhibition of specific immune responses by feeding protein antigens. IV. Evidence for tolerance and specific active suppression of cell-mediated immune responses to ovalbumin. J. Immunol. 123: 2344-2351.

32. Bourguin, I., Chardes, T., Mevelec, M. N., Woodman, J. P. and Bout, D. 1991. Amplification of the secretory IgA response to Toxoplasma gondii using cholera toxin. FEMS Microbiol. Lett. 81: 265-272.

33. Anastassiou, E. D., Yamada, H., Francis, M. L., Mond, J. J. and Toskos, G. C. 1990. Effects of cholera toxin on human B cells: Cholera toxin induces B cell surface DR expression while it inhibits anti- $u$ antibody-induced cell proliferation. J. Immunol. 145: 2375-2380.

34. Francis, M. L., Moss, J., Fritz, T. A. and Mond, J. J. 1990. cAMP-independent effects of cholera toxin on B cell activation. I. A possible role for cell surface ganglioside $\mathrm{G}_{\mathrm{m} 1}$ in $\mathrm{B}$ cell activation. J. Immunol. 145: 31623169.

35. Lycke, N. E., Severinson, E. and Strober, W. 1990. Cholera toxin acts synergistically with IL-4 to promote IgG1 switch differentiation. J. Immunol. 145: 3316-3324.

36. Kim, D. K., Nau, G. J., Lanki, D. W., Dawson, G. and Fitch, F. W. 1988. Cholera toxin discriminates between murine $\mathrm{T}$ lymphocyte proliferation stimulated by activators of protein kinase $\mathrm{C}$ and proliferation stimulation by IL-2. Possible role for intracellular cAMP. J. Immunol. 141: 3429-3437. 
This document is a scanned copy of a printed document. No warranty is given about the accuracy of the copy. Users should refer to the original published version of the material. 